\title{
Formar ciudadanos desde el pre-escolar
}

\section{Sandra León-Muñoz}

Pontificia Universidad Javeriana, Colombia

salem@javerianacali.edu.co
José J. Kenji López-Takegami

Pontificia Universidad Javeriana, Colombia

\section{Resumen}

Se presenta una revisión de los lineamientos educativos para la formación en competencias ciudadanas en el preescolar en Colombia, asi como de investigaciones sobre el desarrollo cognitivo-social en el niño preescolar, estableciendo relaciones con los programas de intervención en competencias ciudadanas en estas edades. Se revisaron en total 38 estudios. La discusión se enfocó en la necesaria articulación que se debe dar entre los avances investigativos en el desarrollo cognitivo-social del niño y los programas de intervención en competencias ciudadanas en niños de preescolar, con el fin de desplegar acciones ciudadanas, defensivas y propositivas en esta etapa del ciclo vital, pertinentes para que sean utilizadas por los educadores.

\section{Palabras clave}

Educación preescolar, pedagogía infantil, desarrollo cognitivo social, competencias ciudadanas (Fuente: Tesauro de la Unesco).

Recepción: 2014-10-27 | Envío a pares: 2015-02-10 | Aceptación por pares: 2015-08-06 | Aprobación: 2015-08-10 DOI: 10.5294/edu.2015.18.2.4

Para citar este artículo / To reference this article / Para citar este artigo

León-Muñoz, S., López-Takegami, J. K. (2015). Formar ciudadanos desde el pre-escolar. Educ. Educ., vol. 18, No. 2, 245-260. DOI: 10.5294/ edu.2015.18.2.4 


\title{
Forming Citizens as of the Pre-School Level
}

\begin{abstract}
This article reviews a series of educational guidelines for training in citizenship skills at the preschool level in Colombia, along with research on cognitive and social development in preschool children. It also takes a look at how these guidelines and findings relate to programs for teaching citizenship skills to youngsters in this age group. Thirty-eight studies were reviewed. The discussion focused on the articulation that is required between progress in research on children's cognitive and social development and programs to teach citizenship skills to preschool youngsters. The objective was to provide an idea of civic, defensive and purposeful actions that are pertinent and can be used by teachers at this stage in a child's life.
\end{abstract}

\section{Keywords}

Preschool education, child pedagogy, cognitive and social development, citizenship skills (Source: Unesco Thesaurus). 


\section{Formar cidadãos desde a pré-escola}

Resumo

Apresenta-se uma revisão dos lineamentos educativos para a formação em competências cidadãs na pré-escola na Colômbia, bem como de pesquisas sobre o desenvolvimento cognitivo-social da criança em idade pré-escolar estabelecendo relações com os programas de intervenção em competências cidadãs nessa faixa-etária. Revisaram-se, em total, 38 estudos. A discussão foi enfocada na necessária articulação que deve ocorrer entre os avanços na pesquisa em desenvolvimento cognitivo-social da criança e os programas de intervenção em competências cidadãs em crianças de pré-escola a fim de realizar ações cidadãs, defensivas e propositivas nessa etapa do ciclo vital, pertinentes para que sejam utilizadas pelos educadores.

\section{Palavras chave}

Educação pré-escolar, pedagogia infantil, desenvolvimento cognitivo-social, competências cidadãs. (Fonte: Tesauro da Unesco). 


\section{Introducción}

La ciudadanía se entiende como una condición social y política que enmarca el reconocimiento de los hábitos, las costumbres y los valores de una sociedad específica; el ejercicio de derechos civiles, sociales y políticos; el cumplimiento de deberes correlativos a tales derechos; y la capacidad de aportar, constructivamente, al desarrollo de la sociedad (Díaz, 2004). Entre los derechos civiles consagrados en la Constitución Política de Colombia de 1991 se encuentra la libertad, la igualdad y la dignidad. Entre los derechos sociales sobresalen los relativos al trabajo, la salud y la educación; y entre los derechos políticos se encuentra la posibilidad de elegir y ser elegido. Los derechos civiles y sociales, en Colombia, pueden ser exigidos y ejercidos desde que la persona nace, mientras que algunos derechos políticos, como votar, pueden ejercerse a partir de los 18 años.

Así, el ejercicio de la ciudadanía, desde su concepción política, civil y social, implica la ejecución de dos tipos de acciones: defensivas y propositivas. Las primeras involucran una defensa y exigencia de derechos, defensa de los derechos de otros, entre otras. Las acciones propositivas hacen referencia a la participación en reformas para cambiar normas, reglas y procedimientos para proponer o sostener relaciones y modificar condiciones de vida injustas, indignas y/o violentas, dentro de la sociedad, en bien de todos sus miembros (Ruiz y Chaux, 2005).

Adicional a lo anterior, la ciudadanía, como condición política, implica también un acatamiento y un ejercicio, entendido como acción en lo público, por parte del ciudadano (Ruiz y Chaux, 2005). El acatamiento alude a la conciencia que tienen los miembros de la sociedad de pertenecer a un orden social regido por normas, valores y costumbres y a la capacidad que tienen para reconocer los derechos y deberes que poseen dentro de la sociedad. Por tanto, para asumir la formación de ciudadanos desde el preescolar es importante conocer los aportes que los estudios sobre el desarrollo cognitivo-social, en esta etapa del ciclo vital, han realizado sobre temas como la construcción de normas, la empatía, la conducta prosocial, el reconocimiento de emociones, entre otros.

Asumiendo lo anterior, este artículo presenta una revisión de estudios sobre el desarrollo cognitivo-social en el niño preescolar y sobre los programas en formación ciudadana que implican acciones defensivas, entendidas como aquellas que involucran la exigencia de derechos, y acciones propositivas, asumidas como aquellas que buscan bienestar para todos los miembros del entorno. Interesa conocer cómo son asumidas estas acciones en los programas de intervención en competencias ciudadanas en niños en preescolar y explorar las relaciones que se articulan entre estos y los resultados de las investigaciones en desarrollo cognitivosocial en el niño preescolar.

\section{Formar ciudadanos desde el preescolar}

Para lograr un ejercicio pleno de la ciudadanía, actuando defensiva y propositivamente, una persona debe alcanzar, por un lado, el dominio gradual de conocimientos concernientes a derechos, deberes, mecanismos de participación y conocimientos acerca del funcionamiento de la sociedad y del Estado relativo a normas, leyes y políticas (Dudley y Curtina, 2008; Díaz, 2004); y, por otro lado, desde los procesos psicológicos involucrados, requiere que la persona desarrolle y despliegue habilidades cognitivas, emocionales y comunicativas, para relacionarse con otros, resolver conflictos y sostener la convivencia en la sociedad. El dominio de los conocimientos y habilidades mencionadas se logra en la interacción con otros miembros de la sociedad y con instituciones sociales como la familia y la escuela, contextos ideales para formar en ciudadanía desde edades tempranas (Díaz, 2000).

En cuanto a la formación ciudadana en el preescolar, programas nacionales e internacionales aluden a la importancia de que se enseñe en los currículos diseñados en las escuelas conocimientos y 
habilidades dirigidos a que los niños alcancen una comprensión de las implicaciones de ser ciudadano, y puedan así realizar un adecuado ejercicio de su ciudadanía dentro de una comunidad específica. Se plantea entonces que aprender a ser ciudadano no es algo que ocurra al alcanzar una edad adulta, sino que se construye desde edades tempranas y se consolida a lo largo del ciclo de vida.

En la literatura científica revisada, los autores afirman que es posible que los niños preescolares "logren" una comprensión del significado de ciudadanía (Covell, Howe y McNail, 2008). Estos estudios sugieren que las escuelas incluyan en sus currículos programas para incentivar en los alumnos: 1) conocimientos y comprensión de lo que significa: tener derechos, hacerlos respetar, valorar los de otros y cumplir con sus responsabilidades dentro del contexto escolar, familiar y social en general; 2) valores como el respeto y la justicia en la interacción con otras personas; 3 ) aprendizajes relacionados con hacer valer los propios derechos dentro del contexto escolar, sin transgredir los de sus pares, con el fin de sostener relaciones interpersonales entre pares y con adultos; y 4) habilidades que les permitan actuar acorde con las exigencias de la sociedad civil y entender las políticas que la rigen, al igual que las relaciones de poder que en ella se establecen (Covell, Howe y Mcnail, 2008; Howard y Gill, 2000; Johansson, 2009).

Ahora bien, el Ministerio de Educación Nacional (MEN) ha promulgado políticas públicas dirigidas a promover la formación ciudadana desde la educación preescolar hasta la educación secundaria, prescribiendo que tal formación debe enfocarse, primordialmente, en el desarrollo de competencias ciudadanas en niños y jóvenes. Tales competencias son descritas como el conjunto de conocimientos, actitudes y habilidades cognitivas, emocionales y comunicativas que permiten a los ciudadanos entender y participar en las interacciones ciudadanas, así como intervenir constructivamente en una sociedad democrática y orientar mo- ral y políticamente su acción ciudadana (Mahecha, 2006; MEN, 2005; Ruiz y Chaux, 2005).

Para hacer realidad la política anterior, de acuerdo con el MEN (2005) y Ruiz y Chaux (2005), se deben abordar los siguientes cuatro tipos de competencias ciudadanas. Las competencias cognitivas entendidas como aquellas disposiciones mentales que se ponen en acción para facilitar el buen ejercicio de la ciudadanía. En este punto, sobresalen habilidades como el reconocimiento de la perspectiva de los demás, la interpretación de las intenciones en los demás, el juicio moral, la resolución de problemas y el pensamiento crítico. Las competencias emocionales, respecto de las cuales se mencionan habilidades como la identificación y regulación de las propias emociones y la atribución y comprensión de emociones en los demás. Las competencias comunicativas, que consisten en las disposiciones de las personas para comprender la realidad simbólica construida socialmente; aquí sobresalen habilidades como la escucha activa y la asertividad, que facilitan la expresión de ideas y el intercambio de conocimientos entre las personas. Finalmente, Ruiz y Chaux (2005) hacen referencia a las competencias integradoras, que involucran el despliegue, en conjunto, de las competencias cognitivas, comunicativas y emocionales. Un ejemplo de esta última competencia consiste en tomar decisiones morales que se caractericen por mediar entre los intereses propios y los de los demás.

En sintesis, desde este punto de vista, la formación en competencias ciudadanas en preescolar debe fomentar las acciones ciudadanas que apunten a respetar al otro, solidarizarse con él, cumplir los deberes, resolver conflictos, garantizar la convivencia pacífica y la paz, y a la participación ciudadana, la pluralidad, la valoración y el respeto de las diferencias dentro de la sociedad, todo lo cual involucra la realización tanto de acciones defensivas como de acciones propositivas. Sin embargo, los imperativos de la política y este catálogo del "deber ser" para formar ciudadanos desde el preescolar se 
deben relacionar con los aportes de los estudios en desarrollo cognitivo-social del niño en preescolar para poder precisar el qué, definir el cómo y conocer el cuándo dentro de esta etapa del ciclo vital.

\section{Desarrollo cognitivo-social en el niño preescolar}

Formar ciudadanos desde el preescolar implica entonces, tanto para educadores como para todos los diferentes profesionales que están liderando este propósito, conocer y discutir los resultados que han mostrado los estudios sobre la construcción del mundo social en el niño y, con los resultados en este dominio de conocimiento, conocer los funcionamientos que tienen los niños y las relaciones que establecen, para comprender el mundo social en el que están inmersos, para interactuar con otros dentro de este mundo y para llevar a cabo acciones prosociales, de respeto y de solidaridad con los demás.

A este campo de estudio se lo ha denominado desarrollo cognitivo-social. Este campo permite establecer cómo los niños comienzan a construir representaciones, desplegar las habilidades mentales y construir relaciones interpersonales para comprender las implicaciones de estar en un medio social, actuar acorde a sus reglas y exigencias y desarrollar acciones propositivas y defensivas en la construcción de ciudadanía.

Para autores como Flavell (1996) y Perinat (1998), el conocimiento social se entiende como un conocimiento psicológico que implica, en primer lugar, la representación de percepciones, intenciones y emociones, respecto de sí mismo y de las otras personas; en segundo lugar, la comprensión y uso de las reglas que controlan las interacciones con los demás, que se aplican a los acuerdos sociales presentes en la interacción que se establece con el otro; y, por último, la comprensión del funcionamiento de las organizaciones sociales.

Por su parte, autores como Turiel (1997) consideran tres contenidos del conocimiento social: 1) los objetos sociales, es decir, las personas; 2) las organizaciones en las que las personas participan (familia, escuela, gobiernos, empresas); 3) las vivencias interpersonales que se relacionan con la justicia y orientan hacia el conocimiento moral. Al respecto, Perinat (1998) plantea que el conocimiento social comienza a construirse desde edades tempranas en torno a personas, interacciones, instituciones y reglas.

Para la construcción del conocimiento social es necesario que el niño pueda establecer interacciones duraderas con otras personas: niños, jóvenes, adultos y adultos mayores, y que interactúe y valore las primeras organizaciones sociales, que se suponen aliadas en su proceso de desarrollo, como lo son la familia y la escuela.

Por tanto, con relación a la interacción con otras personas, en los trabajos con niños en preescolar es importante considerar los desarrollos de una línea de trabajo en el campo cognitivo-social, la cual se centra en el concepto de teoría de la mente, que considera la capacidad de los seres humanos para establecer representaciones mentales acerca de intenciones, deseos, creencias, emociones, respecto de sí mismo y de los demás, que dan lugar a la existencia de un sistema conceptual fundamental para la comprensión de otras maneras de conocimiento y formas de regular las interacciones sociales (Riviére y Núñez, 1996; Ziv y Frye, 2004).

A partir de las investigaciones en teoría de la mente se conoce que desde temprana edad los niños pequeños adquieran habilidades básicas para reconocerse y reconocer al otro, dentro de un entorno que demanda una constante interacción con familias y comunidades (Wellman y Lagattuta, 2004). Así, desde el descubrimiento que hace el niño de la mente le posibilita ser consciente de que tanto él como los otros poseen estados mentales y que dichos estados guían sus acciones (Rodríguez, García, Gorriz y Regal, 2001; Benavides y Roncancio, 2009). Dentro de los estudios de teoría de la mente se encuentran tres tendencias teóricas explicativas 
de cómo los niños llegan a comprender y atribuir estados psicológicos: la teoría-teoría, la teoría de la simulación y la teoría modular (Flavell y Miller, 1998; Benavides y Roncancio, 2009). Esta revisión se centró en la teoría-teoría.

Para la teoría- teoría, los niños desarrollan una serie de hipótesis que emplean para interpretar y explicar el mundo. A medida que los niños se enfrentan al contexto social estas teorías explicativas se modifican y les permiten reajustarlas. Dentro de esta postura, la mente del niño es vista como un sistema conceptual, sistema que se evidencia en la comprensión y atribución de estados psicológicos a otros y a sí mismo, desde edades muy tempranas, incluso desde el nacimiento (Flavell y Miller, 1998).

En la postura teoría-teoría se evidencia el interés por reconocer en los niños aspectos como deseos, intenciones, creencias y emociones, en busca de ver cómo responden a estos y a diversas situaciones a las cuales se encuentran vinculados. Dentro del marco de interpretación de la teoría de la mente, una tarea permite establecer criterios de creencias limitadas a una situación específica, lo cual hace posible desarrollar la noción perceptiva y el reconocimiento de cómo se adquiere el conocimiento (Miller, 2000, citado por Ziv y Frye, 2004).

En esta misma línea, Wimmer y Perner (1983, citados en Riviére y Núñez, 1996) estudiaron el paradigma de la falsa creencia, el cual consistía en presentar al niño un elemento que había sido puesto en un sitio X por el personaje A, elemento que al salir A de la habitación es cambiado del lugar $X$ al $Y$ por el personaje B. Cuando A regresa a la habitación, a quien se le cuenta la historia se le pregunta por el lugar en el que A buscará el elemento guardado. Esta tarea exige al niño la representación de su propia creencia, precisar en qué lugar se encuentra el elemento guardado, así como la representación del posible cambio de lugar. Con esta capacidad representacional se comienza a evidenciar que desde los cuatro años los niños perciben las creencias de las otras personas. A la edad de tres años se evidencia cómo, a pesar de comprender la relevancia de los personajes de una historia, los elementos que intervienen y la historia en general, los niños vinculan su propia realidad y no la realidad a la que ha estado expuesto un personaje en la historia (Flavell y Miller, 1998; Riviére y Núñez, 1996; Ziv y Frye, 2004).

Ziv y Frye (2003) plantean que cuando la relación entre acción, deseo y creencia se desarrolla en la falsa creencia, como pasa con el cambio de ubicación de un objeto deseado por el niño pero del cual no conoce su nueva ubicación, "el niño desarrolla un sistema conceptual e inferencial (teoría de la mente) que sirve a la vez, tanto para predecir y explicar la conducta ajena, como para dar cuenta de la propia" (Wimmer y Hartl, 1991; Sullivan y Winner, 1991, citados en Riviére y Núñez, 1996, p. 36).

\section{Teoría de la mente y comprensión de emociones}

Las emociones y los afectos son considerados como los pilares de la teoría de la mente, por tanto, han sido establecidos como un camino inicial para acceder a la intersubjetividad. Autores como Trevearthen (1982) y Hubley (1978) (citados en Riviére y Núñez, 1996) afirman que la manera de aproximarse a las intenciones de los demás se origina de manera innata en cada individuo. Flavell y Miller (1998) plantean que las emociones deben ser consideradas objeto del pensamiento y de las experiencias sentidas por los niños. Para los anteriores autores, los niños desde los dos años de edad comienzan a evidenciar estados emocionales a través de expresiones como: triste, enojado, feliz. Afirman que los niños pequeños comprenden las emociones al relacionar situación y acción y que estas relaciones se consolidan en la escuela cuando adquieren conceptos más elaborados sobre las emociones.

Para Harris (1989) la comprensión de emociones se encuentra ligada a la capacidad de los sujetos de interpretar no solo la expresión facial que acom- 
paña la emoción, sino las distintas situaciones que las suscitan. La comprensión de emociones es entonces una función cognitiva soportada en la interacción social, la cual implica concebir las relaciones sociales y contextuales en las cuales se encuentran inmersos los sujetos, teniendo en cuenta: los actores involucrados y la interacción entre los autores y las normas sociales implicadas en dichas interacciones. Dicha comprensión de las relaciones permite a los sujetos comprender la conducta propia y ajena y predecir, ocultar, promover y manipular las emociones y por consiguiente las relaciones con los demás (Perinat, 1998).

Denham (2003) encontró que cuando los niños preescolares expresan emociones como la alegría, la tristeza y la rabia, estas tienen un impacto en la manera como establecen las relaciones con sus pares. En cuanto a la comprensión y conocimiento de emociones propias y ajenas, muestran comprensión de los contextos en los que ocurren las emociones y las consecuencias que pueden tener. La comprensión emocional entonces aporta a la interaccion social que los niños construyan.

Es claro también que el contexto escolar donde está inmerso el niño juega un papel importante en la comprension emocional, dado que las relaciones entre el educador y los niños y las relaciones de los niños entre ellos influyen en el desarrollo cognitivosocial de estos y determinan la manera como se van a comportar y, en últimas, las acciones que realizaran en el espacio de convivencia que comparten: el aula. Por tanto, se hace necesario generar pautas para que los educadores de los niños acompañen el desarrollo emocional del niño y potencien sus inmensas capacidades de socializar y de generar empatía hacia los demás, de modo que les permitan desplegar habilidades emocionales y sociales adecuadas para ampliar su comprensión y su actuación en el mundo social (Denham, 2003).

En esta vía, Harris (1989) plantea que los niños desarrollan la comprensión emocional a partir de diferentes acciones e interpretaciones sobre las situaciones diarias. Para el autor, los niños realizan primero una identificación de la situación, lo cual conduce al desencadenamiento de emociones de diferente valencia. Esta primera identificación y, más aún, la conciencia de la emoción suscitada llevan al niño a buscar explicaciones mediante el recuerdo de sucesos análogos o similares.

Este desarrollo progresivo de la comprensión emocional lo describe Harris en nueve niveles de comprensión emocional de acuerdo con la expresión facial, las causas externas, los deseos, las creencias, la influencia de un recuerdo en un estado emocional actual, la posibilidad de controlar un estado emocional, la posibilidad de ocultar una experiencia emocional, emociones contradictorias y emociones sociales-morales.

Estos niveles son progresivos y se adquieren, según los autores, a medida que los sujetos comprenden las situaciones en las cuales se generan las emociones y que son capaces de reconocer cómo estas situaciones afectan los estados cognitivos, específicamente, emocionales de las personas. Por tanto, de acuerdo con lo anterior, en el niño en preescolar, la comprensión de las emociones sociales-morales viene antecedida por la construcción de otros niveles de comprensión de emociones, los cuales se deben tener en cuenta en el momento de desarrollar acciones tendientes a la formación de ciudadanos.

En este sentido, es importante considerar que, si bien los estudios revisados se centran en la teoría de la mente, este último nivel, referido a la comprensión de las emociones sociales-morales, debería articularse a los resultados que arrojan los estudios en desarrollo moral, en el sentido que lo plantea Elorrieta-Grimalt (2012) de pasar de las comprensiones y los juicios a las conductas o acciones morales, en clara alusión a los investigadores pos Kohlberg. De igual forma, es importante tener en cuenta que, para la formación de ciudadanos, es necesario trabajar en la comprensión de las emociones del niño 
en preescolar, pero no suficiente; por tanto, dado el momento del ciclo de vida, es preciso considerar también cómo va construyendo el niño la comprensión de deseos y creencias.

\section{Teoría de la mente y comprensión de deseos y creencias}

Las habilidades de los niños para comprender a otros los transforman. Cuando se es consciente de que las acciones son gobernadas por estados intelectuales, como las creencias, los deseos y las intenciones, las relaciones interpersonales se modifican. La teoría de la mente plantea como principio fundamental una triada de creencias, deseos y acciones, aunque se resalta la primacía de la creencia sobre el deseo, ya que la creencia prevalece, mientras que el deseo puede cumplirse o no. De esta manera, en relación con la comprensión de un paradigma, hay diferencia en las edades por su relación entre el deseo y la creencia, en el contexto de la falsa creencia (Moses y Flavell, 1990; Wellman, 1991, citados por Ziv y Frye, 2003).

La adquisición de una teoría de la mente por el niño es el reconocimiento de que las creencias se involucran en la construcción del mundo social del cual el niño hace parte. El conocimiento de la falsa creencia se consigue típicamente alrededor de los cuatro años de edad (Wellman, Cross y Watson, 2001; Wimmer y Perner, 1983; citados en Hughes et al., 2005). Las diferencias individuales en la comprensión de la falsa creencia pueden estar relacionadas con algunos aspectos importantes de las relaciones sociales tempranas.

En pro de predecir la acción, se asocia el deseo y la creencia. Fodor (1992, citado por Ziv y Frye, 2003) establece que los niños de cinco años de edad y mayores incorporan estas dos nociones en función de la predicción de la acción. En los niños de tres años dominan principalmente los deseos, de manera que presentan dificultad para establecer un criterio acerca de la falsa creencia.
Flavell y Moses (citados por Ziv y Frye, 2003) establecen que la mayor dificultad para los niños de tres años está en no diferenciar entre los objetos deseables y no deseables presentes, y en esto coinciden los distintos estudios realizados, los cuales buscan principalmente dar cuenta de las diferencias significativas que impone la edad en el desarrollo de la teoría de la mente. Así, los niños de dos años comprenden solamente los deseos y todavía tienen dificultades en reconocer la creencia; por contraste, los niños de tres años están en un periodo de transición entre la razón y el deseo. Durante este período, los niños empiezan a comprender las creencias, pero la concepción de las creencias es todavía la sombra del deseo (Ziv y Frye, 2003).

En las tareas de falsa creencia los niños pronostican dónde buscará un personaje un objeto cuya ubicación se cambia durante su ausencia. Los niños entre cuatro y cinco años establecen que el personaje buscará el objeto en la ubicación inicial, mientras que los más de tres años establecen como punto de búsqueda la ubicación real del objeto (Amsterlaw y Wellman, 2006).

Moses y Flavell (1990) plantean la importancia de la comprensión de los deseos y las creencias por parte de los niños. Para estos autores la teoría de la mente implica una triada de creencias, deseos y acciones; por ello la mayor dificultad que encuentran en el desarrollo de los niños pequeños es la de diferenciar entre objetos deseables y no deseables presentes y las implicaciones de esto en la comprensión de la mente del otro.

En el plano de la comprensión de deseos, creencias e intenciones, otras investigaciones han sugerido que los niños preescolares pueden comprender los deseos de otras personas y ser capaces de tener presentes sus preferencias al relacionarse con ellos, siempre y cuando hayan alcanzado una comprensión de las preferencias y deseos propios (Atance, Bélanger y Meltzoff, 2010). En la misma línea, el estudio de Moore, Leanne y Macgillivray (2011) sugiere que los 
niños, para establecer relaciones sociales con pares, tienen en cuenta las preferencias, juegos y acciones de estos.

Esta comprensión se da como un proceso evolutivo de la mente, para llegar de manera progresiva a una "explicación (mecanismo mental) de la relación funcional de los estados mentales con el mundo" (Perner, 1994, p. 268). Esta compresión depende de la constitución del sistema representacional en los niños a partir de tres niveles que dan cuenta de las representaciones de los niños. El primer nivel corresponde a las representaciones primarias, las cuales aparecen desde el primer año de vida y refieren a la capacidad de establecer constructos teóricos, tales como la observación. El segundo nivel es el de las representaciones secundarias, que se evidencian aproximadamente a los dos años, cuando los niños son capaces de relacionar lo observado con sus propias vivencias. El tercer y último nivel es el de las metarrepresentaciones, que hacen referencia a la capacidad de comprender aspectos como la intencionalidad y las falsas creencias. En este nivel las meta-representaciones son concebidas como una relación entre las diversas representaciones constituidas por los sujetos a partir de la experiencia (Harris, 1989). Por tanto, es en este último nivel donde el educador debe estar atento al proceso de socialización que el niño está desarrollando, ya que es a partir de la experiencia del encuentro con otros como el niño preescolar evidencia la construcción de intencionalidad en él y en los otros, aspecto fundamental para elaborar escenarios de convivencia y respeto desde preescolar.

\section{Teoría de la mente y reconocimiento de la perspectiva del otro}

El reconocimiento de la perspectiva del otro se concibe como la capacidad que tienen los sujetos para registrar e interpretar los estados mentales de sí mismo y de otros. Esta capacidad es la base del establecimiento de fenómenos psicológicos como la empatía, el autoconocimiento, la conducta proso- cial, las habilidades de comunicación y el desarrollo moral (Abello, Amar y Magendzo, 2006).

La toma de perspectiva es entonces un constructo cognitivo-social que encierra la capacidad para: relacionar dos o más elementos a la vez; comprender el punto de vista de los otros y el propio, situándose en la perspectiva social; comprender que los puntos de vista de un sujeto están en relación con los de otro; diferenciar varios puntos de vista y saber evaluar los datos perceptual y cognitivamente; inferir las características de los sujetos, que implica la descentración; controlar y relativizar el propio punto de vista a la hora de juzgar el punto de vista de los demás (Abello, Amar y Magendzo, 2006).

Una tendencia de los estudios en la perspectiva del otro se centra en establecer cómo el conocimiento de sí mismo llega a ser un objeto de experiencia, cuando es posible observarlo, desde el conocimiento que tenemos de los otros. Esta formación del concepto de sí mismo se desarrolló a partir de la vivencia de experiencias e intercambios sociales. Para Mead (citado en Abello, Amar y Magendzo, 2006) el proceso cognitivo de ponerse en el lugar del otro o hacer inferencias acerca de las experiencias en las que el niño se encuentra inmerso involucra la conceptualización propia de los aspectos de interacción de los sujetos. Desde la cognición social, esta habilidad cognitiva se constituye cuando el niño va logrando tomar conciencia de la existencia de múltiples perspectivas y se va distanciando de la propia para entender el mundo.

Abello (2000) investigó la toma de perspectiva y sostiene ella que es fundamental para la comprensión del comportamiento de los demás, de forma que está relacionada con varios tipos de comportamiento social. El dominio de la toma de perspectiva del conocimiento social está ligado a la interacción social, pero se aprende en la experiencia social. Este autor caracterizó la toma de perspectiva en niños de tres a siete años de la Costa Caribe colombiana (Barranquilla, Santa Marta y Sincelejo) pertene- 
cientes a un contexto vulnerable (estratos 1 y 2) en una muestra de 268 niños adscrito al programa de hogares comunitarios del Instituto Colombiano de Bienestar Familiar (ICBF). Abello tuvo en cuenta la toma de perspectiva cognitiva y comunicativa como componentes de la toma de perspectiva conceptual. Adicionalmente, en su estudio consideró la toma de perspectiva emocional. Definió la toma de perceptiva conceptual como el proceso cognitivo de ponerse en el lugar de los demás y realizar inferencias acerca de lo que piensan o sienten. La toma de perspectiva cognitiva fue asumida como el proceso cognitivo de inferir lo que las demás personas saben o no de sí mismas o de las demás. Fue evaluada por medio de dos historias graficadas en las que los niños debían inferir lo que los demás sabían de la historia con un conocimiento limitado de la misma, así como la justificación de sus inferencias.

Mientras que la toma de perspectiva comunicativa se entendió como la habilidad del emisor de tener en cuenta al receptor cuando este le dice algo. Se estudió a partir de un juego de escogencia, en el cual consideraron el número de verbalizaciones adecuadas que realizaban los niños para escuchar la elección de un juguete por parte de otra persona que tenía los ojos vendados. Finalmente, en ese estudio se asumió la toma de perspectiva emocional como el proceso cognitivo de hacer inferencias acerca de los estados emocionales de los demás. Se utilizaron una serie de tarjetas y en cada una se presentaba una situación en la que dos personajes expresaban emociones distintas. Se preguntaba a los niños si comprendían las emociones de dichos personajes.

Los resultados de Abello (2000) sugieren que los niños de este contexto presentan mejor desempeño en la toma de perspectiva emocional que en la conceptual. Para el autor la explicación del mejor desempeño de los niños en la toma de perspectiva emocional se debe a fortalezas del contexto. Dentro de las ventajas del contexto, el autor identifica la evidencia de valores como la solidaridad, la identidad grupal, la comprensión de sentimientos y la conducta prosocial. El autor identifica como causa de los bajos resultados en la toma de perspectiva cognitiva la pobreza del contexto educativo, ya que no propicia espacios prácticos que les permitan a los niños recrear conflictos sociocognitivos propios de su contexto, para que a partir de ellos tengan experiencias que promuevan su construcción social de la realidad.

Autores como Fishbein, Lewis y Keiffer (1972, citados en Abello, Amar y Magendzo, 2006) han realizado estudios centrados en la representación del punto de vista de los otros. Para ello, pedían a niños de tres años y medio que eligieran el dibujo que mejor representaba el punto de vista del otro, frente a una situación planteada (presentación de un carro de bomberos de juguete) entre un grupo de dibujos. Los resultados permitían observar cómo los pequeños lograban anticipar lo que el otro iba a realizar o estaba pensando de la situación presentada.

Por otra parte, autores como Arenas (2007), Paulus y Moore (2011) y Olson y Spelke (2008) han estudiado cómo los niños se representan las relaciones con lo demás y en qué condiciones realizan acciones cooperativas con ellos, de lo cual infieren: a) que los niños tienden a compartir más con familiares o amigos que con extraños y a la reciprocidad ante acciones cooperativas de otros y a recompensarlos, si han ayudado a alguien; b) que la cooperación y la conducta altruista entre niños pequeños están mediadas por la capacidad que tengan estos de comprender y predecir estados mentales en el otro; y c) que pedir ayuda e involucrarse en conductas de cooperación en los niños también parece estar mediado por la comprensión que los niños tengan de las capacidades de los demás para realizar ciertas acciones.

El papel de la toma de perspectiva es entonces cognitivo-social y exige a los sujetos inferir capacidades, atributos, expectativas, sentimientos y reacciones potenciales, que comienzan a evidenciar desde los tres o cuatro años de edad (Aznar, Pérez y Sánchez, 1990). 
Las investigaciones centradas en la toma de perspectiva han permitido la elaboración de sistemas de categorizacion que responden al desarrollo de esta capacidad cognitiva a lo largo de la vida. Dentro de estos sistemas se encuentran el elaborado por Flavell (1996) que responde a tres niveles de toma de perspectiva, donde el nivel 1 corresponde al reconocimiento de los motivos de la elección de otro, sin reconocer que el otro conoce los motivos propios. El nivel 2 corresponde al reconocimiento de los pensamientos, elecciones y acciones del otro, percatándose de la capacidad de ese otro para comprender sus pensamientos, elecciones y acciones. Y el nivel 3, que implica una toma de perspectiva mutua, donde cada sujeto (yo mismo y el otro) comprende y considera sus perspectivas y las del otro (Cabrerizo, Fernández, Lozano y Núñez, 2001).

Selman (1971, citado por Aznar, Pérez y Sánchez, 1990) señala, por su parte, cinco niveles evolutivos en el desarrollo del reconocimiento de la perspectiva del otro: El nivel o corresponde a las edades entre tres y seis años, cuando existe indiferenciación entre los deseos y creencias propias y ajenas y se piensa que el otro ve lo mismo que uno. En el nivel 1 los niños en edades entre cinco y nueve años comprenden que el punto de vista propio difiere del punto de vista del otro y que cada sujeto percibe las situaciones de diferente manera. En el nivel 2 los niños entre siete y doce años comprenden que los pensamientos y sentimientos de una persona son múltiples y que la adopción de perspectiva es autorreflexiva y recíproca. En el nivel 3 los niños entre diez y quince años ya son capaces de coordinar simultáneamente las perspectivas propias y las de otros. En el nivel 4, desde los doce años en adelante, los sujetos tienen conciencia de lo relativo y complejo de las situaciones sociales y de la elaboración de múltiples perspectivas frente a estas.

En síntesis, con relación al desarrollo cognitivo-social, se encontraron estudios que consideraron la comprensión de emociones que construye el niño preescolar, tanto de sus emociones como de las ajenas; y estudios sobre teoría de la mente especialmente centrados en la comprensión de deseos y creencia; así como estudios en el reconocimiento de la perspectiva del otro y en las conductas prosociales.

Los anteriores temas del desarrollo cognitivosocial en el niño en preescolar se consideran importantes para abordar el diseño de los programas de intervención en formación ciudadana con preescolares y permiten precisar, desde aspectos concretos que involucran el desarrollo cognitivo-social del niño en preescolar, las cuatro competencias que conforman la formación ciudadana propuesta por el Ministerio de Educación Nacional.

\section{Programas de Intervención en Formación Ciudadana del niño preescolar}

Con relación a los programas de intervención se encontró que algunos, como el programa Aulas en Paz, dirigido a niños hasta quinto de primaria, tuvieron como objetivo promover en los escolares el desarrollo de competencias ciudadanas como la empatía, la toma de perspectiva, la regulación de emociones, la generación de alternativas de solución y valoración de sus consecuencias, la escucha activa, la asertividad y el pensamiento crítico. En Aulas en Paz se retoman resultados de los estudios en desarrollo cognitivo-social en el niño, pero se deja por fuera la competencia integradora entre la escuela y la familia. (Chaux, 2009).

Otros programas de formación ciudadana se plantean como objetivo impulsar la formación ética y ciudadana en niños y jóvenes y propusieron como ejes centrales la enseñanza de: a) principios democráticos, como la participación en la toma de decisiones y la mediación en resolución de conflictos; c) derechos humanos; y d) herramientas para fomentar la inclusión social y luchar contra la discriminación en la sociedad. Araújo y Arantes (2009) plantean, más que soluciones, interrogantes sobre el cómo de la enseñabilidad de los aspectos referidos. 
En este mismo sentido, se encontró otro programa de intervención en formación ciudadana, el cual involucraba diferentes agentes, como profesores, padres de familia y organizaciones sociales y estatales. Deseando aportar a la formación de ciudadanos activos y constructivos, haciendo énfasis en el desarrollo de competencias críticas que les permitan identificar necesidades y dificultades de su contexto social, para proponer cambios que logren impulsar el mejoramiento de dicho contexto, en beneficio de todos sus miembros. Y, por último, los agentes involucrados en la formación ciudadana debían procurar que los niños y jóvenes pusieran en práctica en su experiencia cotidiana valores como la solidaridad y facilitaran entre ellos el intercambio y la comprensión de ideas, el diálogo, la participación y el establecimiento de acuerdos para la toma de decisiones (Oraisón y Pérez, 2009). No obstante que este programa involucra otros agentes sociales en su propuesta de formación ciudadana, el educador de niños en preescolar continúa sin saber cómo realizar las articulaciones entre estos agentes.

En otro sentido, Marmolejo y Jiménez (2005), retomando resultados de estudios en cognición social, proponen realizar la formación ciudadana en niños en preescolar desde la lectura de textos narrativos, puesto que en este ejercicio el niño debe entender la situación en la que está el personaje y las emociones y pensamientos que le suscita dicha situación, así como anticipar decisiones y eventos y reconocer la perspectiva de otros en la situación. De esta forma, se pueden crear historias que representen situaciones sociales que impliquen las habilidades relacionadas con la ciudadanía, donde se logre evidenciar lo que el niño puede hacer, lo que sabe y lo que necesita aprender para la comprensión de la ciudadanía y así sentar las bases para su ejercicio actual y futuro. Por otro lado, León (2006) propone enfatizar el trabajo con niños en preescolar desde la lectura de imágenes. Y a pesar de que investigadores, educadores y padres reconocen la importancia de la lectura de textos narrativos, las preguntas que emergen son, de nuevo, por la factibilidad de ello en los escenarios reales que afronta el educador de preescolares, en aulas con 25-30 niños y por la articulación del uso de esta técnica entre la escuela y la familia.

Hasta este punto se tiene claro, entonces, que la formación ciudadana en el preescolar es un tema de interés tanto para organismos educativos estatales como el Ministerio de Educación Nacional como para investigadores que abordan el desarrollo cognitivo-social en el niño preescolar.

No obstante lo anterior, comprendidas las políticas y los lineamientos nacionales y entendiendo que diversos estudios en desarrollo cognitivo-social se plantean desde metodologías experimentales o no experimentales, donde se estudian grupos selectos o muestras precisas de niños, abordados en muchas ocasiones en estudios transversales, la pregunta para el educador que día a día se enfrenta o afronta el reto de acompañar los procesos de desarrollo social de niños en preescolar sigue siendo el cómo.

Creemos que los estudios en desarrollo cognitivo-social han permitido ampliar el qué, y mostrar esto fue la intención de uno de los apartados anteriores; sin embargo, en la cotidianidad de cualquier contexto escolar la complejidad y la convergencia de los diversos sistemas sociales son imperativos. Por tanto, con relación a los programas de intervención en formación ciudadana, dos de los desafíos que se presentan para investigadores y educadores son: 1) ¿cómo construir espacios, acciones, tareas, situaciones que tengan en cuenta los aportes de los estudios en desarrollo cognitivo-social y los lineamientos del MEN, en contextos escolares reales de educación pública que albergan en sus aulas entre 25 y 30 niños preescolares, algunos con necesidades educativas especiales (estudiantes NED)?; y 2) ¿cómo articular las acciones que los educadores desempeñan en el aula con los procesos de educación continua que se deben dar en las familias de estos niños, es decir, cómo construir corresponsabilidades entre escuela y familia para formar ciudadanos desde el preescolar? 
La articulación de los resultados de los estudios en desarrollo cognitivo-social con niños en preescolar en los programas de intervención en formación ciudadana implica la diferenciación entre la formación ciudadana y el concepto de competencia, pues creemos que la formación conlleva la integración de acciones en un sentido integrativo y relacional. Por ello se defiende la necesidad de involucrar en los programas de intervención a la familia, ya que el concepto de competencia obedece a un análisis individual de desempeño de los sujetos, a veces en escenarios no naturales o frente a tareas descontextualizadas que no consideran las relaciones cotidianas que se entretejen con el contexto donde el sujeto, en este caso el niño en preescolar, vive, y donde sigue faltando el cómo para la construcción de escenarios de convivencia, en los que está siempre en relación con alguien, no en una actuación solitaria o en una actitud solo mentalista.

Adicional a lo anterior, la articulación de la investigación en desarrollo cognitivo-social a los programas de formación ciudadana en preescolares implica que se amplíen las fuentes de información en los diseños de los estudios, pues, como lo plantean Ramírez y Arcila (2013), los reportes de estudios en contextos educativos toman como fuente de información a los estudiantes y siempre en una menor proporción se tiene como fuente de información a educadores, padres de familia y comunidad educativa (constituida por estudiantes, educadores, directivos, equipos de orientación escolar, tutores), además de cuidadores "alternos", como la persona encargada de la alimentación en la institución escolar, ya que todos hacen parte del mundo social del niño preescolar.

Así, con relación a la formación ciudadana en el niño preescolar, la pregunta por cómo construir y mejorar la calidad de las relaciones interpersonales que se tejen en los contextos donde se educan a los niños está abierta a quienes se interesan en investigar en primera infancia, frente a la cual siguen esperando respuestas los educadores que acompañan el día a día del desarrollo de los niños en esta etapa del ciclo vital.

\section{Referencias}

Abello, R. (2000). Infancia y conocimiento social. Investigación con niños que viven en contextos de pobreza. Colombia: Uninorte.

Abello, R., Amar, J. y Magendzo, S. (2006). Toma de perspectiva en niños pobres en Colombia. Revista Infancia, Adolescencia y Familia, 1, 189-201.

Amsterlaw, J.y Wellman, H. (2006). Theories of mind in transition: A microgenetic study of development of false belief understanding. Journal of cognition and development, 7 (2) 139-172.

Araújo, U. y Arantes, A. (2009). The ethics and citizenship program: a Brazilian experience in moral education. Journal of Moral Education, 38 (4), 489-511.

Arenas, M. (2007). Habilidades mentalistas y conducta prosocial en niños de edad escolar con características de deprivación social. Revista Corporeizando, 1 (1), 1-32.

Atance, C., Bélanger, M. y Meltzoff, A. (2010). Preschoolers' Understanding of Others’ Desires: Fulfilling Mine Enhances My Understanding of Yours. Developmental Psychology, 46 (6), 1505-1513.

Aznar, P., Pérez, P.y Sánchez, F. (1990). Intervención educativa en el proceso de role-taking, desarrollo de un programa de acción. Revista de Educación, 307-311. 
Benavides, J. y Roncancio, M. (2009). Conceptos de desarrollo en estudios sobre teoría de la mente en las últimas tres décadas. Avances de Psicología, 27 (2), 297-310.

Cabrerizo Fernández, P., Fernández de Vega, T., Lozano Guerra, I.y Núñez del Río, C. (2001). Distinción mente y realidad: una tarea de toma de perspectiva conceptual en una población especial. Revista Española de Pedagogía, 9 (219), 331-346.

Chaux, E. (2009). Citizenship competencies in the midst of a violent political conflict: The Colombian educational response. Harvard Educational Review, 79 (1), 84-167.

Chaux, E., Lleras, J. y Velázquez, A. (eds.) (2004). Competencias ciudadanas de los estándares al aula. Bogotá: Uniandes.

Covell, K., Howe, B.y McNeil, J. (2008). “If there's a dead rat, don't leave it”. Young children's understanding of their citizenship rights and responsibilities. Cambridge Journal of Education, 38 (3), 321-339.

Denham, S. (2003). Preschool emotional competence pathway to social competence? Child Development, $74(1), 238-256$.

Díaz, A. (2000). La participación de las niñas y los niños y la formación ciudadana. Sincronía, 32, 70-82.

Díaz, A. (2004). Desarrollo humano, socialización y formación de ciudadanía desde el preescolar. Perspectivas, 6, 25-33.

Dudley, N. M. y Cortina, J. M. (2008). Knowledge and skills that facilitate the personal dimension of citizenship. Journal of Applied Psychology, 93 (6), 1249-1270.

Elorrieta Grimalt, M. P. (2012). Análisis crítico de la educación moral según Lawrence Kohlberg. Educación y Educadores, 15 (3), 497-512.

Flavell, J. (1996). El desarrollo cognitivo. España: Prentice Hall

Flavell, J. y Miller, P. (1998). Social cognition. Cognition, Perception and Language, 2, 851-898.

Harris, P. (1989). Children and emotion. The development of psychological understanding. New York: Basil Blackwell.

Howard, S. y Gill, J. (2000). The pebble in the pond: Children's construction of power, politics and democratic citizenship. Cambridge Journal of Education, 30 (3), 357-378.

Hughes, C. et al. (2005). Origins of individual differences in theory of mind: from nature to nurture? Child development, 76 (2), 356-370.

Johansson, E. (2009). The preschool child of today -the world citizen tomorrow. International Journal of Early Childhood, 41 (2), 79-95.

León Muñoz, S. (2006). Narrativas orales y lectura de imágenes en niños pre-escolares. Pensamiento Psicológico, 2 (7), 113-131.

Mahecha, M., Díaz, C., Espinoza, G., González, H. y Salcedo, J. (2006). La formación ciudadana de niños, niñas y jóvenes. Revista Actualidades Pedagógicas, 49, 67-79. 
ISSN $0123-1294$ | Educ.Educ. Vol. 18. No. 2 | Mayo-Agosto de 2015 | pp. 245-260.

Universidad de La Sabana | Facultad de Educación

Marmolejo, F. y Jiménez, A. (2005). La formación de competencias ciudadanas en el preescolar. Apuntes de Psicología, 23 (2), 219-226.

Ministerio de Educación Nacional - MEN (2005). ICFES. Programa Saber. Evaluación Censal. Bogotá: MEN. Recuperado el 15 de junio de 2010 de: http://www.colombiaaprende.edu.co/html/home/1592/articles-89525_ archivo.pdf

Ministerio de Educación Nacional - MEN (2009). Guía operativa para la presentación del servicio de atención integral a la primera infancia. Recuperado el 15 de junio de 2010 de: http://www.mineducacion.gov.co/ primerainfancia/1739/articles-178515_archivo_pdf_ruta_version_marzo_2009.pdf

Moore, Ch., Leanne, S. y Macgillivray, S. (2011). Theory of mind an social interest in zero acquaintance play situations. Child Development, 82 (4), 1163-1772.

Moses, L. y Flavell, J. (1990). Inferring false beliefs from actions and reactions. Child Development, 61, 929-945.

Olson, K. R.y Spelke, E. S. (2008). Foundations of cooperation in young children. Cognition, 108, 222-231.

Oraisón, M. y Pérez, A. (2009). The school in non-inclusive context; moral education, building citizenship and community development, an Argentine example. Journal of Moral Education, 38 (4), 515-532.

Paulus, M. y Moore, C. (2011). Whom to ask for help? Children's developing understanding of other people' action capabilities. Experimental Brain Research, 211, 593-600.

Perinat, A. (1998). El conocimiento social. La teoría de la mente. En Psicología del desarrollo: un enfoque sistémico (pp. 259-268). Barcelona: Universitat Oberta de Catalunya.

Perner, J. (1994). Comprender la mente representacional. Barcelona: Paidós.

Ramírez López, C. A. y Arcila Rodríguez, W. O. (2013). Violencia, conflicto y agresividad en el escenario escolar. Educación y Educadores, 16 (3), 411-429.

Riviére, A. y Núñez, M. (1996). La mirada mental. Buenos Aires: AIQUUE.

Rodríguez, N., García, E., Gorriz, A. y Regal, R. (2001). ¿Cómo se estudia el desarrollo de la mente? Universitat Jaume, 1-13.

Ruiz, D. C. y Chaux, E. (2005). La formación de competencias ciudadanas. Bogotá. Ascofade. Recuperado el 15 de junio de 2010 de: http://redes-cepalcala.org/inspector/DOCUMENTOS\%2OY\%2OLIBROS/COMPETENCIAS /LA\%2OFORMACION\%2ODE\%2OCOMPETENCIAS\%2OCIUDADANAS.pdf

Turiel, E. (1997). El mundo social en la mente infantil. Madrid: Alianza.

Wellman, H. y Lagattuta, K. (2004). Theory of mind for learning and teaching: the nature and role of explanation. Cognitive Development, 19 (4), 479-497.

Ziv, M. y Frye, D. (2003). The relation between desire and false belief in children's theory of mind: no satisfaction? Developmental Psychology, 39 (5), 859-876.

Ziv, M. y Frye, D. (2004). Children's understanding of teaching: the role of knowledge and belief. Cognitive Development, 19 (4), 457-477. 